\title{
O que ocupa os médicos de família? Caracterização do trabalho médico para além da consulta
}

Mónica Granja,* Carla Ponte**

\section{RESUMO}

Objectivos: Determinar o tempo despendido pelos médicos de família com consultas e com tarefas para além da consulta, o número de consultas e de contactos não presenciais e o tipo de tarefas realizadas.

Tipo de estudo: Exploratório, observacional, descritivo, transversal.

Local: Centros de saúde da Unidade Local de Saúde de Matosinhos (ULSM).

População: Médicos de família (MF) da ULSM.

Métodos: Convidou-se uma amostra de conveniência de 46 MF a registar, durante 2 a 5 dias, nos meses de Junho e Julho 2010 : tempos de permanência na sua unidade, de pausa e de realização de tarefas para além da consulta; número de contactos presenciais e não presenciais; e tipos de tarefas realizadas. A amostra foi caracterizada e as variáveis foram codificadas, registadas e tratadas informaticamente usando parâmetros de estatística descritiva.

Resultados: Participaram 13 MF (taxa de resposta 28\%) e registaram-se as actividades de 52 dias, incluindo 986 consultas, 514 contactos não presenciais e 101 horas de tarefas. Do tempo de trabalho efectivo diário, $23 \%$ foi ocupado com tarefas (média diária 01h:57m), com registo de uma média de 10 contactos não presenciais, e 06h:31m foram dedicadas a uma média de 19 consultas por MF. As tarefas mais reportadas foram: telefonemas/e-mails, registos, avaliação de resultados de exames, renovação de prescrições, emissão de relatórios/declarações, contacto com outros profissionais, referenciações e reuniões de serviço. Conclusões: O tempo diário despendido pelos médicos de família em tarefas para além da consulta foi 01h:57m, correspondendo a $23 \%$ da sua carga horária e aproximando-se do encontrado em estudos norte-americanos. Na interpretação destes resultados há a considerar possíveis vieses de selecção e de informação. Este estudo torna visível a multiplicidade de tarefas que os MF realizam e o tempo com elas despendido, o que poderá contribuir para um maior reconhecimento do seu peso e importância e a uma maior satisfação profissional.

Palavras-chave: Prática Profissional; Medicina Geral e Familiar; Carga de Trabalho.

\section{INTRODUÇÃO}

endo a consulta a pedra basilar do exercício da Medicina Geral e Familiar (MGF), tem vindo a ser progressivamente assumido que as tarefas que competem aos médicos de família (MF) se estendem muito para além do encontro entre médico e paciente no consultório. Nestas tarefas para além da consulta incluem-se as relativas a pacientes (quer decorrendo de uma

* Médica de Família, Centro de Saúde Senhora da Hora, Unidade Local de Saúde de Matosinhos.

**Médica de Família, Unidade de Saúde Familiar Porta do Sol, Unidade Local de Saúde de Matosinhos. consulta recente, quer sem relação com esta) e as não relacionadas com pacientes específicos (ou não clínicas).

As tarefas que decorrem da própria consulta (como é o caso de registos, referenciações ou contactos com outros profissionais) podem ter de ser diferidas da mesma pela pressão assistencial, por falhas no sistema informático ou pela indisponibilidade no momento da consulta de outros profissionais com quem seja necessário articular.

As tarefas relativas a pacientes previamente conhecidos mas não presentes para consulta são múltiplas e incluem, entre outras, a renovação de medicação crónica (da qual decorreu a criação, há uma década, do conceito de «contacto 
não presencial»), a avaliação e comunicação de resultados de exames complementares de diagnóstico, os contactos telefónicos e, mais recentemente, as mensagens de correio electrónico.

Estes dois últimos meios têm sido expressamente recomendados para ampliar a acessibilidade e continuidade de cuidados, quer internacionalmente, ,1,2,3 quer na MGF portuguesa, ${ }^{4}$ quer mesmo na Unidade Local de Saúde de Matosinhos (ULSM). ${ }^{5}$

Quanto às tarefas designadas como não clínicas, algumas, como aquelas relacionadas com a recolha e análise de indicadores, a participação nas actividades de planeamento do serviço, a colaboração na formação e educação médicas e, mesmo, a participação em projectos de investigação, possuem já moldura legal enquanto competência dos médicos em geral. ${ }^{6,7}$

Outras tarefas que podemos designar como não clínicas incluem as que consistem em suprir insuficiências do sistema e levam, não raro na experiência das autoras, a que MF assumam competências que não as suas (como é o exemplo do aprovisionamento de material clínico e de escritório ou da dispensa de contraceptivos).

O interesse pela descrição e quantificação do tempo despendido nas tarefas para além da consulta prende-se com três factores distintos, embora relacionados entre si: a retribuição ligada ao desempenho que tem ganho terreno na MGF; a dificuldade em registar parte destas tarefas; $\mathrm{e}$ a invisibilidade deste trabalho, que põe em risco a existência de tempo protegido para a sua realização e acarreta insatisfação profissional.

\section{A REMUNERAÇÃO LIGADA AO DESEMPENHO}

Em Portugal, com a Reforma dos Cuidados de Saúde Primários, a metodologia de contratualização em funcionamento desde 2006 já contempla incentivos institucionais às Unidades de Saúde Familiares (USF) que cumpram indicadores de desempenho pré-definidos., ${ }^{8,9}$

No entanto, os indicadores de desempenho apresentam limitações importantes como o facto de serem medidas de proximidade, serem dependentes de registos (sem se acompanharem de sistemas de informação adequados) e não representarem as especificidades da MGF. ${ }^{10}$

A sua utilização exclusiva para avaliação do desempenho do MF leva a uma visão reduzida das suas verdadeiras competências, sobrevalorizando aspectos de registo em detrimento das qualidades primordiais da especialidade: abordagem bio-psico-social da pessoa, gestão de problemas múltiplos, promoção de saúde e coordenação e integração de cuidados.

A descrição da actividade do MF deve ser também ampla, para abranger todas as facetas do seu desempenho. Existem, assim, inúmeras tarefas realizadas pelos MF que, sendo essenciais para atingir o nível de qualidade desejada e garantir a satisfação dos utentes, não são contabilizadas na sua produtividade e não se reflectem na sua remuneração.

\section{OS REGISTOS CLÍNICOS}

A aplicação informática usada para registo electrónico pela maioria dos MF portugueses, o SAM ${ }^{\circledR}$ (Sistema de Apoio ao Médico), só permite a inserção de notas clínicas depois de um registo administrativo de contacto que só pode ser efectuado pelos profissionais administrativos no programaSINUS ${ }^{\circledR}$. Isto inviabiliza que sejam registados telefonemas, e-mails e contactos através de familiares ou cuidadores em que apenas se troquem informações, seja fornecido aconselhamento ou dado retorno quanto a resultados complementares de diagnóstico.

Perturbando a continuidade de cuidados e acarretando um risco clínico apreciável, a falta de registo deste tipo de elementos clínicos é inclusive proscrita pelo Conselho Médico-legal. ${ }^{11}$

A falta de registos torna também estas tarefas, de todas, as mais invisíveis, e, como tal, muito difíceis de medir, avaliar e gerir.

\section{A INVISIBILIDADE}

Vários estudos sobre a adesão de médicos ao uso do correio electrónico apuraram que um dos obstáculos ao seu uso era o facto de ser uma tarefa não contabilizada em termos de produtividade..$^{12-15}$ Mesmo na ausência de remunerações dependentes da produtividade, o reconhecimento inter-pares e pelos órgãos de gestão é um factor de satisfação profissional, a qual, por sua vez, é uma das determinantes da qualidade dos cuidados prestados. ${ }^{16}$

Por outro lado, a recente reformulação das carreiras médicas e respectivas condições contratuais dos MF portugueses, ${ }^{17,18}$ embora inclua no perfil do MF uma multitude de atribuições muito além da mera realização de consultas, não contempla tempo protegido nos horários médicos para a realização destas tarefas. Este tempo protegido era contemplado na legislação que previamente regulava as carreiras médicas (Lei 73/90), sendo designado como «horas destinadas a funções não assistenciais», tendo sido, em 
2000, tacitamente estendido aos primeiros MF com contratos individuais de trabalho. No entanto, no contexto actual de escassez de MF e controlo progressivamente mais apertado dos horários e agendas médicas, torna-se cada vez mais difícil que órgãos de gestão aloquem tempo protegido para a realização de tarefas que sejam invisíveis (isto é, que não estejam descritas nem quantificadas em termos de carga horária) e há já notícias de situações em que foram estabelecidos horários integralmente preenchidos com consultas, sem que fosse alocado qualquer tempo para a realização de tarefas para além da consulta. ${ }^{19}$

Não foram encontrados em Portugal dados estatísticos nem estudos publicados sobre o tipo e quantidade de tarefas que os MF desempenham para além da consulta. Da pesquisa bibliográfica efectuada, obtiveram-se apenas quatro estudos (todos eles norte-americanos e realizados nos últimos 5 anos) avaliando as tarefas além da consulta de médicos em contexto de cuidados primários. Todos os estudos revelaram que estas tarefas constituem uma parte importante do trabalho médico. ${ }^{20-23}$

Assim, realizou-se um estudo com os seguintes objectivos:

- quantificar o tempo despendido pelos MF com consultas e com tarefas para além da consulta;

- determinar o número de consultas e de contactos não presenciais realizados nos dias do estudo;

- descrever as diferentes tarefas desempenhadas pelos MF para além da consulta.

\section{MÉTODOS}

Realizou-se um estudo exploratório, observacional, descritivo e transversal, sobre uma amostra não aleatória de conveniência de MF de seis unidades de saúde da ULSM, durante os meses de Junho e Julho. À data do início estudo, a ULSM contava com 18 unidades de saúde (6 USF, 7 UCSP e 5 de outro tipo) onde estavam colocados $103 \mathrm{MF}$ (dados dos Recursos Humanos da ULSM), os quais constituíram a população em estudo. ${ }^{1}$ Foram convidados a participar os 46 MF de quatro USF (Horizonte, Lagoa, Maresia e Porta do Sol) e de 2 UCSP (Sra. da Hora e Leça da Palmeira). O convite foi enviado por $e$-mail dirigido aos coordenadores das respectivas unidades e a cada MF, tendo também sido posteriormente feitos pelas investigadoras contactos pessoais e por telefone. Àqueles que se mostraram interessados foi enviado o protocolo do estudo e, após confirmação da participação, pedida a assinatura de um texto de consentimento informado. Após a manifestação da vontade em participar, foram feitos contactos periódicos com cada participante até à entrega de todos os dados necessários.

Pediu-se a cada MF que escolhesse para a colheita dos dados entre 2 a 5 dias úteis de trabalho consecutivos dos meses de Junho e Julho, de modo a não incluir duas vezes o mesmo dia da semana. Sempre que não foi possível a inclusão de cinco dias úteis consecutivos de trabalho, foi pedido que o período total de registo correspondesse a um mínimo de 14 horas de trabalho na unidade de saúde.

Foi contabilizado o tempo despendido com todas as tarefas que foram desempenhadas pelos MF participantes na unidade de saúde para além da consulta, assinalando-se numa grelha a hora de início e de fim da tarefa em causa (observada em relógio digital e com aproximação ao minuto). Foram também determinadas quais as diferentes tarefas que os MF realizam para além da consulta, discriminadas segundo uma taxonomia desenvolvida pelas autoras a partir dos quatro estudos congéneres realizados e que, depois da realização de um estudo piloto, foi adaptada à realidade dos locais de trabalho das autoras. ${ }^{20-23} \mathrm{O}$ estudo piloto foi realizado pelas autoras durante um dia típico de trabalho. Os dados foram colhidos por cada um dos MF participantes, registados em tempo real numa grelha desenvolvida para o efeito e de acordo com um guião previamente distribuído, sendo posteriormente codificados e tratados pelas autoras.

Para cada MF e em cada dia de trabalho estudado, foi ainda contabilizado: o tempo total de permanência na unidade de saúde; os tempos de pausa e de actividades pessoais; o número total de registos administrativos de contacto e, destes, o número de não presenciais; a presença na consulta de internos ou de estudantes; e o grau de tipicidade do dia de trabalho (usando uma escala de Likert onde 1 será um dia totalmente típico e 5 um dia totalmente atípico).

\footnotetext{
${ }^{1}$ Optou-se por não incluir na amostra cinco unidades de saúde atípicas (duas relacionadas com a assistência a grupos profissionais específicos e três clínicas privadas que funcionam ao abrigo do projecto «Medicina em Concorrência», criado pela portaria n. ${ }^{\circ}$ 667/90 de 13 de Agosto). Das restantes foram inicialmente escolhidas cinco unidades, das quais duas eram UCSP e três eram USF. No entanto, já depois de iniciados os contactos, uma das UCSP dividiu-se, originando uma UCSP e uma USF, totalizando seis unidades participantes. As cinco unidades inicialmente escolhidas foram seleccionadas por critérios de exequibilidade: três por maior proximidade física (importante na fase de recrutamento) ao local de trabalho das duas investigadoras; uma por ser à data a única USF modelo B da ULSM para além daquela onde trabalha uma das investigadoras; e uma outra que, pela sua maior dimensão (posteriormente dividida em duas unidades), rentabilizava melhor os contactos a maior distância.
} 
Foram ainda recolhidas as seguintes variáveis de caracterização dos MF participantes: sexo, idade, anos de actividade com especialista de MGF, dimensão da lista de utentes, funções como orientador de formação do internato da especialidade, local de trabalho e características contratuais.

Foi inicialmente prevista a recolha de dados sobre o tempo despendido com cada tipo de tarefas, mas o estudo piloto realizado mostrou que não era viável a colheita desses dados pelo próprio $\mathrm{MF}$, sobretudo devido à frequente realização de tarefas diferentes, quer em simultâneo, quer intercaladas em curtos espaços de tempo, o que inviabilizava a colheita em tempo real.

As variáveis foram codificadas, registadas e tratadas em suporte informático (Microsoft Excel ${ }^{\circledR}$ ). Foram determinados os parâmetros para caracterização dos MF, calculado o tempo despendido com tarefas realizadas para além da consulta e descritas as diferentes tarefas realizadas. A amostra foi caracterizada e comparada com os dados disponíveis relativos à população. Foram listadas as diferentes tarefas realizadas pelos MF para além da consulta. As restantes variáveis foram analisadas usando métodos de estatística descritiva. Todos os valores relativos a tempos foram colhidos e contabilizados arredondados ao minuto.

\section{RESULTADOS}

Dos $46 \mathrm{MF}$ convidados a colaborar, 13 (incluindo as duas autoras) aceitaram participar no estudo (taxa de resposta 28,3\%), pertencentes a 4 (3 USF e 1 UCSP) das 6 unidades convidadas e representando $12,6 \%$ da população de MF da ULSM.

Os MF participantes tinham em média 37 anos e apenas dois $(15,4 \%)$ são do género masculino. Em média, os MF participantes haviam terminado a sua especialidade há 6,5 anos, sendo que apenas um era especialista há mais de 10 anos. Dos treze, doze estão vinculados à ULSM por um contrato individual de trabalho, onze trabalhavam em USF à data do estudo (oito dos quais em modelo B). Os MF que não estavam em modelo B tinham horário semanal de 40 horas, acrescendo num dos casos duas horas extra semanais numa base fixa.

Dos MF participantes, cinco eram orientadores de formação do internato de Medicina Geral e Familiar (21,7\% dos OF da ULSM) e, destes, três estavam a trabalhar com internos em nove dos 52 dias de trabalho estudados. Um outro MF teve consigo um estudante em três dos 52 dias do estudo.
Adimensão média das listas dos MF participantes era de 1710 utentes. Nos quadros I e II são representadas as características demográficas e profissionais dos MF participantes, assim como as dos não respondentes.

Os MF da amostra que não aceitaram participar no estudo (não respondentes) eram, em média, mais velhos (44.1 vs 36.6 anos), trabalhavam como MF há mais tempo (14.2 vs 6.5 anos), eram mais frequentemente do género feminino (90.9 vs $84.6 \%$ ) e contavam com uma menor proporção de orientadores de formação do internato de MGF (26 vs $38,5 \%)$. Aplicando os testes $\chi^{2}$ e $t$ de Student, estas diferenças entre os dois grupos (respondentes e não respondentes) obtiveram significância estatística $(p<0,05)$. A proporção de MF a trabalhar em USF foi maior no grupo dos respondentes (72,7 vs $84,6 \%$ ), diferença também estatisticamente significativa. Não foi encontrada diferença estatisticamente significativa entre respondentes e não respondentes apenas quanto à dimensão média da lista de utentes.

\section{Tempo despendido com tarefas para além da consulta}

Os treze MF participantes registaram as actividades de um total de 52 dias de trabalho (média de quatro dias por participante, mínimo 2, máximo5), correspondendo a mais de 455 horas, que incluíram 986 consultas, 514 contactos não presenciais e 101 horas dedicadas às tarefas para além da consulta.

Foi ocupado na realização de tarefas para além da consulta $23 \%$ do tempo de trabalho efectivo diário, correspondendo a uma média de 1 hora e 57 minutos. Em média, cada MF permaneceu diariamente na sua unidade de saúde 8 horas e 46 minutos, das quais, 8 horas e 25 minutos foram reportadas como de trabalho efectivo. Seis horas e 31 minutos de trabalho diário foram dedicadas à realização de uma média de 19 consultas por MF, calculando-se, para cada consulta, uma média de 22 minutos. Os resultados sobre o tempo de trabalho estão representados no quadro III.

Nos cinquenta e dois dias estudados, além das consultas realizadas, foram ainda registados administrativamente uma média de 10 contactos não presenciais diários por MF participante, resultando numa proporção de contactos presenciais/não presenciais de cerca de 2:1. Dividindo o número de contactos não presenciais registados administrativamente pelo total de tempo despendido com tarefas para além da consulta, a cada contacto não presencial corresponde uma média de 13 minutos (quadro IV).

Numa escala (de Likert) de 1 a 5, em que 1 significava 
muito típico e 5 significava pouco típico, os dias estudados foram classificados pelos MF quanto à sua tipicidade, em média, como 2,2 (quadro IV).

\section{Tipo de tarefas realizadas para além da consulta}

Os vários tipos de tarefas assinalados pelos MF participantes foram sistematizados pelas autoras em dois grandes grupos: as directamente relacionadas com pacientes específicos (designadas como tarefas clínicas) e as não directamente relacionadas com pacientes (designadas como não clínicas). Nos quadros V e VI são discriminadas as diferentes tarefas assinaladas.

A maioria das tarefas foi reportada pela generalidade dos MF participantes no estudo: telefonemas, registos, avaliação de resultados de exames complementares, renovação de prescrições, emissão de relatórios e declarações, interacção com outros profissionais, referenciações e reuniões de serviço. Algumas tarefas foram assinaladas por cerca de metade dos MF: $e$-mails, estudo de lista e de indicadores, paragens devidas a falhas informáticas e reposição ou procura de material de apoio à consulta. Outras ainda foram assinaladas por uma minoria de MF: resolução de conflitos entre profissionais e utentes, espera por doentes atrasados, marcações de consultas, investigação, atendimento de delegados de informação médica, gestão, coordenação e integração de grupos de trabalho, estudo e pesquisa de fontes bibliográficas e organização do consultório e arquivo.

\section{DISCUSSÃO}

O tempo despendido pelos MF em tarefas para além da consulta foi neste estudo estimado em cerca de 2 horas diárias, correspondendo a quase um quarto da sua carga horária. O tipo

DP desvio-padrão

MF médico de família de tarefas desempenhadas foi muito variado, desde as directamente relacionadas com pacientes e transversais a todos os MF do estudo (como a renovação de receituário crónico, a observação e comunicação de resultados de exames complementares de diagnóstico, o contacto directo não

\begin{tabular}{|lccc}
\hline \multicolumn{4}{|c|}{ QUADRO I. Características demográficas e profissionais dos médicos de família } \\
participantes ( $\mathrm{n}=13$ ) e dos não respondentes $(\mathrm{n}=33)$ & - parte 1. \\
& Participantes & Não respondentes & $\boldsymbol{P}$ \\
\hline Idade média (anos) & 36,6 & 44,1 & 0,013 \\
\hline Máximo & 55 & 57 & \\
\hline Mínimo & 28 & 28 & \\
\hline DP & 7,3 & 10,9 & \\
\hline IC 95\% & 3,9 & & \\
\hline Tempo médio como MF (anos) & 6,5 & 14,2 & \\
\hline Máximo & 27 & 28 & \\
\hline Mínimo & 0 & 0 & \\
\hline DP & 7,3 & 11,4 & \\
\hline IC95\% & 4,3 & & \\
\hline Dimensão média lista utentes & 1710 & 1656 & \\
\hline Máximo & 1795 & 1852 & \\
\hline Mínimo & 1502 & 397 & \\
\hline DP & 97,1 & 261 & \\
\hline IC95\% & 47,3 & & \\
\hline
\end{tabular}

IC $95 \%$ intervalo de confiança a $95 \%$

\begin{tabular}{|c|c|c|c|c|}
\hline \multicolumn{5}{|c|}{$\begin{array}{l}\text { QUADRO II. Características demográficas e profissionais dos médicos de } \\
\text { família participantes }(n=13) \text { e dos não respondentes }(n=33) \text { - parte } 2 \text {. }\end{array}$} \\
\hline \multirow[b]{3}{*}{ Género feminino } & \multicolumn{2}{|c|}{ Participantes } & \multirow{3}{*}{$\begin{array}{c}\text { Não respondentes } \\
\qquad \% \\
90,9\end{array}$} & \multirow{3}{*}{$\begin{array}{c}P \\
0,00159\end{array}$} \\
\hline & $\mathrm{n}$ & $\%$ & & \\
\hline & 11 & 84,6 & & \\
\hline Tipo de unidade & & & & 0,00041 \\
\hline UCSP & 2 & 15,4 & 27,3 & \\
\hline USF & 11 & 84,6 & 72,7 & \\
\hline $\begin{array}{l}\text { Orientadores } \\
\text { formação }\end{array}$ & 5 & 38,5 & 26 & 0,00037 \\
\hline
\end{tabular}

UCSP unidade de cuidados de saúde personalizados

USF unidade de saúde familiar 
presencial por telefone ou $e$-mail e a referenciação hospitalar), às que cabem pontualmente a apenas alguns dos MF estudados (como a gestão e coordenação, a investigação e o ensino ou formação).

Um estudo realizado em 2005 numa unidade de saúde norte-americana, no qual onze MF foram observados durante dois dias por estudantes de medicina treinados, encontrou valores um pouco superiores aos do presente estudo: $30,5 \%$ do tempo efectivo de trabalho era ocupado em tarefas para além da consulta e, deste, $23 \%$ era para tarefas clínicas e 7,5\% para tarefas não clínicas. ${ }^{21}$ Noutro estudo semelhante, realizado também em 2005, em unidades de saúde norte-americanas e em que estudantes de Medicina observaram $27 \mathrm{MF}$ durante um dia de consulta, a proporção de tempo de trabalho alocado às tarefas para além da consulta foi também de $23 \%$, mas não incluiu nestas algumas tarefas tais como contactos com pacientes por $e$ mail ou telefone ou activida-

des de ensino e formação. ${ }^{20}$ Apesar de o contexto da prática da MGF norte-americana ser bastante diversa da portuguesa (com os pacientes a serem observados numa sala diferente daquela onde posteriormente o MF faz os registos, emite as credenciais para exames ou prescreve a medicação), ambos os estudos encontraram valores semelhantes aos do presente estudo relativamente ao tempo total de permanência na unidade de saúde e à proporção deste tempo despendido com tarefas para além da consulta..$^{20,21}$

Um outro estudo, realizado em 2007, estimou que cada médico gastava semanalmente em actividades para além da consulta o mesmo que no presente estudo se apurou gastar diariamente, mas, apesar de realizado em ambiente de cuidados primários e assentar sobre registos em tempo real pelo próprio médico, o facto de incidir sobre geriatras de uma unidade de saúde norte-americana não permite com- paração directa entre resultados..$^{22}$

Este mesmo estudo sobre geriatras comunitários estimou uma duração de cerca de 6 minutos por cada contacto não presencial, ${ }^{22}$ enquanto que, no presente estudo, se obteve uma correspondência de 13 minutos por cada contacto não presencial registado administrativamente. Considerando que há tarefas não relacionadas com contactos com pacientes (investigação, reuniões, coordenação, etc.) e que há contactos não presenciais (telefonemas e $\boldsymbol{e}$-mails, por exemplo) que não são registados administrativamente, o tempo real por cada contacto não presencial será seguramente inferior. O não registo de todas as tarefas para além da consulta é um dos determinantes da invisibilidade de componentes essenciais do perfil e da prática do $\mathrm{MF}$ nos quais se despende tempo considerável.

As tarefas para além da consulta descritas no estudos 
QUADRO V. Tarefas directamente relacionadas com utentes realizadas para além da consulta.

\section{TAREFAS RELACIONADAS COM PACIENTES}

Telefonemas de e para utentes, familiares ou cuidadores

E-mail de e para utentes, familiares ou cuidadores

Registos clínicos

Procura, observação, registo e comunicação de resultados de exames complementares de diagnóstico

Renovação de prescrições solicitadas via secretariado, e-mail, telefone ou na consulta de um familiar

Pedidos de exames na sequência de telefonema ou e-mail (inclui segundas vias)

Emissão de relatórios, declarações e atestados

Procura, recepção, leitura, registo de informação clínica de outros profissionais

Comunicação clínica com outros profissionais sobre utentes

Comunicação administrativa com outros profissionais sobre utentes

ALERT, outras referenciações, consultar estado referenciação e resolução de problemas

Estudo/pesquisa de fontes bibliográficas a propósito de utentes

Resolução de conflitos entre profissionais e utentes

Tempo de espera por utentes com atraso

Marcar consultas a pedido de outros profissionais ou de familiares ou cuidadores

disponíveis não diferem substancialmente das encontradas neste estudo, à excepção de: interacções com companhias de seguros (frequentes nos estudos norte-americanos e ausentes neste estudo) e presença nos hospitais e em actividades hospitalares (referidas nos estudos norte-americanos e não indagadas neste por se ter pedido o registo apenas das tarefas realizadas dentro da unidade de saúde). ${ }^{20-23}$ Por outro lado, não há referência nos estudos acedidos a tarefas identificadas neste, como: falhas informáticas, reposição e procura de material de apoio à consulta ou resolução de conflitos.

Resultado acessório deste estudo mas que se salienta por si, é o facto de o tempo médio de trabalho efectivo na unidade de saúde reportado pelos MF ser superior a 8 horas diárias. Considerando que a este tempo de trabalho efectivo na unidade de saúde se somam ainda as consultas ao
QUADRO VI. Tarefas sem relação directa com utentes

realizadas para além da consulta.

\section{TAREFAS NÃO RELACIONADAS COM PACIENTES}

Estudo da lista ou de indicadores, planeamento tarefas relacionadas com indicadores

Tempo de espera e de resolução de falhas informáticas (paragens ou lentidão do sistema)

Repor ou procurar material de escritório ou clínico

Actividades relacionadas com internos ou alunos

Reuniões de serviço ou reuniões clínicas de formação contínua

E-mail de serviço não relacionados com utentes específicos

Telefonemas de serviço não relacionados com utentes específicos

Investigação e tarefas relacionadas

Atendimento delegados de informação médica

Tarefas de gestão ou coordenação do serviço ou integração de grupos de trabalho

Estudo não relacionado com utentes específicos (fontes bibliográficas, orientações técnicas, etc.)

Tarefas de gestão pessoal, arrumação consultório, arquivo, etc.

domicílio, tempos de trabalho para além da consulta na unidade de saúde ao sábado e domingo (referidas por alguns participantes no período do estudo mas não contabilizadas) e várias outras actividades passíveis de serem realizadas fora da unidade de saúde (como as actividades decorrentes da OF), fica claro que as cargas horárias semanais clássicas de 40 horas parecem ser largamente ultrapassadas.

Outro resultado acessório do estudo diz respeito ao número médio de consultas e ao tempo médio gasto por consulta. De referir que, apesar de os MF participantes trabalharem em três modelos diferentes de organização assistencial, com diferentes regras de agendamento, não parece haver grande variação no tempo despendido por consulta (quer programada quer não programada), já que, para um média de 22 minutos, o $D P$ foi de apenas 7 minutos. Na interpretação dos resultados é necessário considerar ainda que os MF orientadores de formação com internos presentes na consulta nos dias do estudo podem ver 0 seu tempo de consulta incrementado (no caso do interno partilhar a consulta com orientador, pela necessidade de in- 
teracção formativa entre ambos), ou artificialmente reduzido (caso o interno faça consulta aos utentes do orientador de forma autónoma mas sendo as consultas contabilizadas como consultas do orientador).

Metodologicamente há a considerar que a técnica de amostragem de conveniência utilizada aportou aos resultados um viés de selecção. A opção por esta técnica de amostragem, considerada aceitável pelas autoras visto tratar-se de um estudo exploratório, foi condicionada pela dispersão física e pela heterogeneidade funcional das 18 unidades de saúde da ULSM, a qual inviabilizaria que as duas autoras pudessem coordenar eficazmente o estudo em todas as unidades.

De igual forma, a taxa de resposta ao convite para participação no estudo, muito inferior à desejável, além de condicionar uma amostra reduzida ( $13 \mathrm{MF}, 12,6 \%$ da população), pode ter aportado um viés de selecção, aceitável pelas características exploratórias do estudo. De facto, a comparação das características profissionais dos MF participantes e dos não respondentes revelou diferenças estatisticamente significativas, sendo os MF estudados mais jovens e mais frequentemente do género masculino, com menos experiência na MGF, contando entre si com mais orientadores de formação e trabalhando mais frequentemente em USF modelo B. No entanto, se, por um lado, os MF que mais tempo despendem neste tipo de actividades poderiam ser os mais motivados à participação; por outro, os MF mais ocupados na generalidade das suas tarefas poderiam ter menos disponibilidade para participar num estudo em que a recolha de dados era mais uma tarefa a acrescer ao seu trabalho diário.

Ainda, sendo a colheita de dados efectuada por cada um dos participantes, são inevitáveis os vieses de informação (pois o registo em tempo real acarreta sempre algum risco de perda de informação) e inter-observador. Este viés poderá atingir com mais importância as variáveis correspondentes à distribuição do tempo de trabalho e ao tipo de tarefas realizado. Pelo contrário, variáveis como o tempo total de permanência na unidade de saúde e o número de contactos presenciais e não presenciais deverão acarretar enviesamento de informação mínimo visto basearem-se em registos informáticos.

A importância principal deste estudo prende-se com o facto de ser um trabalho pioneiro em Portugal, num tema sobre o qual nada existe ainda publicado. Os seus resultados permitiram tornar visível uma multiplicidade de tarefas que os MF realizam para além da consulta e o tempo com elas despendido. Esta visibilidade poderá ser um factor de reconhecimento e satisfação profissional. Poderá também constituir uma oportunidade para que esta significativa parte do trabalho médico possa ser tida em conta pelos responsáveis, quer na planificação da actividade das unidades de saúde, quer na respectiva avaliação de desempenho. Poderá, ainda, alertar para a necessidade de reformular o $\mathrm{SAM} 囚$ de forma a possibilitar o registo sistemático de tarefas para além da consulta.

As autoras têm em curso um projecto para replicar este estudo de forma multicêntrica, recorrendo a estudantes de Medicina como observadores externos, permitindo incluir como objectivos a discriminação do tempo despendido com cada tipo de tarefas e a quantificação das mesmas.

\section{REFERÊNCIAS BIBLIOGRÁFICAS}

1. Committee on Quality Health Care, Institute of Medicine. Crossing the Quality Chasm: a new health system for the 21st century. Washington, DC. National Academy Press; 2001. Disponível em http://books.nap.edu/html/ quality_chasm/reportbrief.pdf [acedido em 2010/12/01].

2. Haggerty JL, Pineault R, Beaulieu MD, Brunelle Y, Gauthier J, Goulet F, et al. Practice features associated with patient-reported accessibility, continuity, and coordination of primary health care. Ann Fam Med 2008 Mar-Apr; 6 (2): 116-23.

3. Gérvas J, Bonis J. El debate profesional acerca de la escasez de médicos. Rev Esp Salud Pública 2008 Nov-Dic; 82 (6): 627-35.

4. Baleiras SJ, Ramos V. A Gestão da Prática Clínica pelo Médico de Família. Rev Port Clin Ger 1992; 9 (4): 116-24.

5. Plano de actividade do ACES Matosinhos 2009.

6. Decreto-Lei n. ${ }^{\circ}$ 176/2009, de 4 de Agosto. Diário da República, $1^{\text {a }}$ série — N. ${ }^{\circ}$ 149. p. 5043-7.

7. Decreto-Lei n. ${ }^{\circ} 177 / 2009$, de 4 de Agosto. Diário da República, $1^{\text {a }}$ série — N. ${ }^{\circ}$ 149. p. 5047-53.

8. Portaria n 301/2008, de 18 de Abril. Diário da República, $1^{\text {a }}$ série - N. ${ }^{\circ} 77$. p. 2278.

9. Ministério da Saúde. Missão para os Cuidados de Saúde Primários. Indicadores de desempenho para as unidades de saúde familiares. Lisboa: Ministério da Saúde; 2006. Disponível em http://www.mcsp.min-saude.pt/Imgs/content/ page_46/Indicadores_desempenho_USF_MCSP20060412.pdf [acedido em 2011/01/16]

10. Santos I, Ribeiro, IL. Indicadores de desempenho na consulta. Rev Port Clin Geral 2009 Mar-Abr; 25 (2): 228-36.

11. Nunes R, Rego G. Questões ético-jurídicas da consulta médica por via telefónica - Parecer do Conselho Médico-legal. Rev Centro Estudos Judiciários 2009; 11: 235-41.

12. Gaster B, Knight CL, DeWitt DE, Sheffield JV, Assefi NP, Buchwald D. Physicians' use of and attitudes toward electronic mail for patient communication. J Gen Intern Med 2003 May; 18 (5): 385-9.

13. Bergmo TS, Wangberg SC. Patients' willingness to pay for electronic communication with their general practitioner. Eur J Health Econ 2007 Jun; 8 (2): 10510.

14. Liederman EM, Lee JC, Baquero VH, Seites PG. Patient-physician web messaging: the impact on message volume and satisfaction. J Gen Intern Med 2005 Jan; 20 (1): 52-7.

15. Car J, SheikhA. Email consultations in health care: 2 - acceptability and safe ap- 
plication. BMJ 2004 Aug 21; 329 (7463): 439-42.

16. Grembowski D, Ulrich CM, Paschane D, Diehr P, Katon W, Martin D, et al. Managed care and primary physician satisfaction. J Am Board Fam Pract 2003 SepOct; 16 (5): 383-93.

17. Acordo Colectivo de Trabalho n²/2009, de 13 de Outubro. Diário da República, 2. a série - N. ${ }^{\circ} 198$. p. 41511.

18. Acordo Colectivo de Trabalho. Boletim do Trabalho e Emprego, $n^{\circ} 41,8 / 11 / 2009$. p. 4496.

19. Reis T. Recém-especialistas da $1^{\text {a }}$ época de 2010 - Assimetrias marcam assinaturas de contratos. Jornal Médico de Família 2010 Nov 24. Disponível em http://www.jmfamilia.com/index.php?option=com_content\&task=view\&id=1 293\&ltemid=27 [acedido em 2010/12/01].

20. Gilchrist V, McCord G, Schrop SL, King BD, McCormick KF, Oprandi AM, et al. Physician activities during time out of the examination room. Ann Fam Med 2005 Nov-Dec; 3 (6): 494-9.

21. GottschalkA, Flocke SA. Time spent in face-to-face patient care and work outside the examination room. Ann Fam Med 2005 Nov-Dec; 3 (6): 488-93.

22. Farber J, SiuA, Bloom P. How much time do physicians spent providing care outside of office visits? Ann Intern Med 2007 Nov; 147 (10): 693-8.

23. Baron RJ.What's keeping us so busy in primary care? A snapshot from one practice. N Engl J Med 2010 Apr 29; 362 (17): 1632-6.

\section{CONFLITOS DE INTERESSE}

As autoras são médicas de família na Unidade Local de Saúde de Matosinhos, local de realização do presente estudo, do qual também foram participantes. Trabalham em duas unidades diferentes:

Mónica Granja trabalha numa Unidade de Cuidados de Saúde Personalizados e, num contrato individual de trabalho de 40 horas, tem uma alocação informal de 4 horas semanais protegidas para desenvolvimento de tarefas para além da consulta.

Carla Ponte trabalha numa Unidade de Saúde Familiar modelo B, sendo uma porção da sua retribuição atribuída por capitação e por desempenho.

Mónica Granja e Carla Ponte tornaram-se membros do corpo editorial da Revista Portuguesa de Clínica Geral após a submissão do presente artigo para publicação, não tendo tomado parte no respectivo processo editorial.

\author{
ENDEREÇO PARA CORRESPONDÊNCIA \\ Mónica Granja \\ Centro de Saúde Sra. da Hora \\ R. Lagoa \\ 4460-352 Senhora da Hora \\ monica.granja@ulsm.min-saude.pt
}

Recebido em 26/01/2011

Aceite para publicação em 27/05/2011

\section{ABSTRACT}

\section{WHAT KEEPS FAMILY PHYSICIANS SO BUSY? A DESCRIPTION OF MEDICAL WORK BEYOND PATIENT ENCOUNTERS}

Objectives: Measuring the amount of time family physicians (FP) spend on direct patient encounters and other medical tasks, determining the number of direct patient encounters and indirect contacts, and describing the range of medical tasks performed.

Design: Observational study.

Setting: Family practices in the Unidade Local de Saúde de Matosinhos (ULSM), Portugal.

Population: Family physicians working in ULSM

Methods: A convenience sample of 46 family physicians was invited to record their activities during 2 to 5 working days, in June and July, 2010. They measured the time spent in practice, on breaks and on medical tasks aside from direct patient encounters, the numbers of direct and indirect patient contacts, and the types of medical tasks performed. The characteristics of the study participants and the characteristics of their tasks were analyzed using descriptive statistics.

Results: Of the 46 family physicians invited, thirteen participated in the study ( $28 \%$ response rate). The activities of 52 working days were recorded, including 986 direct patient encounters, 514 indirect patient contacts and 100 hours of activities. Of the total daily working time, $23 \%$ was spent on non-contact medical tasks (on average one hour and 57 minutes per day), with an average of 10 indirect patient contacts per doctor per day. A mean of six hours and 31 minutes were spent on 19 direct patient contacts per doctor per day. The indirect contact tasks most often reported were: telephone calls, e-mails, medical record keeping, evaluation of laboratory and other tests, prescription refills, completing forms, interactions with other members of the team, referrals and staff meetings.

Conclusions: This sample of family physicians spends an average of one hour and 57 minutes per day on non-contact patient related tasks, accounting for $23 \%$ of their workload. This is similar to the findings from North American studies. There are several likely biases in this study related to sample selection and data collection. This study illustrates the range of tasks that family physicians perform, as well as the amount of time required to perform them. This may contribute to improve the recognition of the workload created by these tasks as well as indicating their importance, with potential effects on professional satisfaction.

Keywords: Professional Practice; Family Practice; Workload. 
has captured our attention and respect, so old style text book articles are rarely accepted or even sent out for review. In the board room we say these articles are typical of the "throwaway" journals that fill our mailboxes. Many of these articles are thinly disguised advertisements for drugs without a truthful statement of conflict of interest by the authors and we don't like that either.

We dislike many of the so-called quality studies that also flooded our inbox for a while. The SQUIRE criteria are an excellent guideline for this kind of work. ${ }^{3}$ Quality projects are a form of research and need to follow the rules of hypothesis generation, sampling, statistical analysis and determination of cause and effect. Studies that don't close the quality loop with an intervention and second look are not interesting and not likely to be published. Perhaps we need to disseminate our guidelines in the classrooms of the quality courses given in the residency schemes around the country. Many of the submissions we receive look like reports of classroom exercises that don't follow the guidelines.

We appreciate the contributions received from hospital specialists or trainees from other disciplines but we hope that they will respect the unique environment in which we work in primary care. Selection bias often affects the case mix and study samples described in their papers. We would hope they would take this into account when making recommendations to family doctors. While we also value the approach and the style of scientific used in other specialities, we often ask ourselves if their manuscripts would be considered for publication or even review in their own speciality journals. The answer is often negative. The relationship between primary care and other specialties is an important area of study and we would welcome more original research on this aspect of health care.

Finally we dislike manuscripts that flagrantly ignore our instructions for authors. Long, disorganized articles with poor spelling and grammar raise immediate questions about the publishable potential of a manuscript. Authors are advised to pay close attention to our guidelines.

\section{WHAT WE WOULD LIKE TO SEE}

We would really like to see a fresh crop of manuscripts, on new topics relevant to general practice that have the potential to improve practice and the health of our patients. We would like to see more research done by the older experienced members of our profession. Most submissions are by trainees. While these are to be encouraged, they lack the polish and perspective of experienced family doctors. We would like to see more interventions and more outcome studies as mentioned above. We would like to see quality improvement studies that really make a difference in practice and are not just running after performance indicators. We want to see critical reviews of existing literature and not just "CV chasing" by eager trainees.

We feel we have taken some important steps forward in the first three issues as an editorial team We look forward to working with you in the next three years to make this an education, enjoyable and innovative journal.

\section{CONFLICT OF INTEREST STATEMENT}

The author is a member of the editorial board of RPCG, a peer-reviewed journal, and has written and reviewed for peer-reviewed journals.

\section{REFERENCES}

1. Yaphe J. Rethinking peer review: an empty slate. Rev Port Clin Geral 2011 May-Jun; 27 (3): 259-60.

2. Hulley SB, Cummings SR, Browner WS, Grady DG, Newman TB. Designing Clinical Research. 3rd ed. Philadelphia, PA: Lippincott, Williams \&Wilkins; 2007. p. 19-22.

3. Heleno B, Pinto D. Para além da avaliação de qualidade. Rev Port Clin Geral 2010 May-Jun; 26 (3): 244-6.

\section{ENDEREÇO PARA CORRESPONDÊNCIA}

yonahyaphe@hotmail.com

\section{CORRECÇÃO}

«O que ocupa os médicos de família? Caracterização do trabalho médico para além da consulta». Neste artigo de Mónica Granja e Carla Ponte (Rev Port Clin Geral 2011;27:388-96) foi, por lapso editorial, omitido o seguinte texto:

As autoras agradecem a colaboração dos médicos de família que participaram no estudo: Dra. Andreia Cerejo, Dr. António Macedo, Dra. Carla Nina, Dra. Carmo Novais, Dra. Ilda Gonçalves, Dr. Jaime Correia de Sousa,
Dra. Joana Santos, Dra. Mariana Tudela, Dra. Raquel Braga, Dra. Sílvia Henriques e Dra. Teresa Pinto Carneiro.

Agradecem ainda ao Dr. Jaime Correia de Sousa pela revisão crítica do manuscrito."

A Revista Portuguesa de Clínica Geral pede desculpas aos médicos de família visados e informa que o texto omitido foi agora incluído na versão on-line do artigo (http://www.apmcg.pt/PageGen.aspx? WMCM_PaginaId=33568\&artId=1074). 